\title{
Trending: Radioactive and Fluorescent Bimodal/Hybrid Tracers as Multiplexing Solutions for Surgical Guidance
}

Fijs W.B. van Leeuwen ${ }^{1,2}$, Margret Schottelius ${ }^{3}$, Oscar R. Brouwer ${ }^{1,2}$, Sergi Vidal-Sicart ${ }^{4}$, Samuel Achilefu ${ }^{5}$, Joachim Klode ${ }^{6}$, Hans-Jurgen Wester ${ }^{4}$, and Tessa Buckle ${ }^{1,2}$

${ }^{I}$ Interventional Molecular Imaging Laboratory, Department of Radiology, Leiden University Medical Center, Leiden, The Netherlands; ${ }^{2}$ Department of Urology, Netherlands Cancer Institute, Antoni van Leeuwenhoek Hospital, Amsterdam, The Netherlands; ${ }^{3}$ Radiopharmaceutical Radiochemistry, Department of Chemistry, TUM School of Medicine, Technical University Munich, Munich, Germany; ${ }^{4}$ Diagnostic Imaging Institute, Hospital Clinic Barcelona, Barcelona, Spain; ${ }^{5}$ Optical Radiology Lab, Department of Radiology, Mallinckrodt Institute of Radiology, Washington University School of Medicine, St. Louis, Missouri; and ${ }^{6}$ Clinic for Dermatology, University Clinic Essen, Essen, Germany

By contributing to noninvasive molecular imaging and radioguided surgery, nuclear medicine has been instrumental in the realization of precision medicine. During the last decade, it has also become apparent that nuclear medicine (e.g., in the form of bimodal/hybrid tracers) can help to empower fluorescenceguided surgery. More specifically, when using hybrid tracers, lesions can be noninvasively identified and localized with a high sensitivity and precision (guided by the radioisotope) and ultimately resected under real-time optical guidance (fluorescent dye). This topical review discusses early clinical successes, preclinical directions, and key aspects that could have an impact on the future of this field.

Key Words: image-guided surgery; fluorescence imaging; nuclear medicine; bimodal tracers; multimodal

J Nucl Med 2020; 61:13-19

DOI: 10.2967/jnumed.119.228684

$\mathbf{W}$ ith regard to the future implementation of imageguided surgery, there is a clear desire to support precision surgery via receptor-targeted molecular imaging. This means that there is a growing need for input from nuclear medicine; next to hybrid modalities such as PET/MRI, combined use of radioactive and fluorescence signals can help strengthen image-guided surgery. This type of image guidance can come in 2 forms. The first is implementation of separate radioactive and fluorescent tracers for pre- and intraoperative imaging. To ensure surgical accuracy, in such a dual-tracer application one has to make sure both tracers independently allow delineation of the same lesions. Although

\footnotetext{
Received Aug. 15, 2019; revision accepted Oct. 22, 2019.

For correspondence or reprints contact: Fijs W.B. van Leeuwen, Interventional Molecular Imaging Laboratory, Department of Radiology, Leiden University Medical Center, Albinusdreef 2, 2300 RC, Leiden, the Netherlands. E-mail: f.w.b.van leeuwen@lumc.nl

Published online Nov. 11, 2019.

COPYRIGHT (C) 2020 by the Society of Nuclear Medicine and Molecular Imaging.
}

such an approach supports the use of existing radiotracers, it is chemically extremely challenging to create fluorescent tracers that behave in an identical manner (on a molecular scale, fluorescent dyes are inherently different from radiolabels). This challenge is exemplified by the sentinel node tracers $99 \mathrm{~m}$ Tc-nanocolloid and indocyanine green (ICG) (1). Second, and the focus of this review, a radioactive and fluorescent signature can be integrated in a single bimodal/ hybrid tracer. Integration ensures colocalization of the two signatures and promotes an advanced form of symbiosis (the best of both worlds) that empowers surgeons to improve intraoperative target delineation (Fig. 1).

Hybrid tracers come in many flavors; not only can the platform on which they are based vary from small molecules $(2,3)$ to nanoparticles (including proteins and nanocolloids (4)), but they also may use different radioisotopes (e.g., $\gamma$ or $\beta+$ emission) or luminescent signatures (e.g., light with different wavelengths; Table 1). Although each individual hybrid tracer and administration route has been designed to serve a specific purpose, conceptually all use revolves around the notion that both signatures can be used to intraoperatively depict complementary features of the same target. Despite differences in signal intensities, there is a high level of overlap in the way multiplexing of the different imaging signatures occurs. In the context of surgical guidance, the radioactive signal allows identification and localization of a lesion by means of its radioactive signature (even in deeper tissue layers), whereas the optical feedback allows direct lesion visualization and delineation in exposed tissue in the surgical field or provides high-resolution pathologic identification of the tracer accumulation (5).

In this review, we discuss where surgical guidance with hybrid tracers stands in relation to fluorescence guidance and radioguidance, describe the early clinical implementation of hybrid tracers, and comment on the preclinical spinoff these efforts have created. Subsequently, we address key requirements in a product-target profile that could support future hybrid tracer development. 


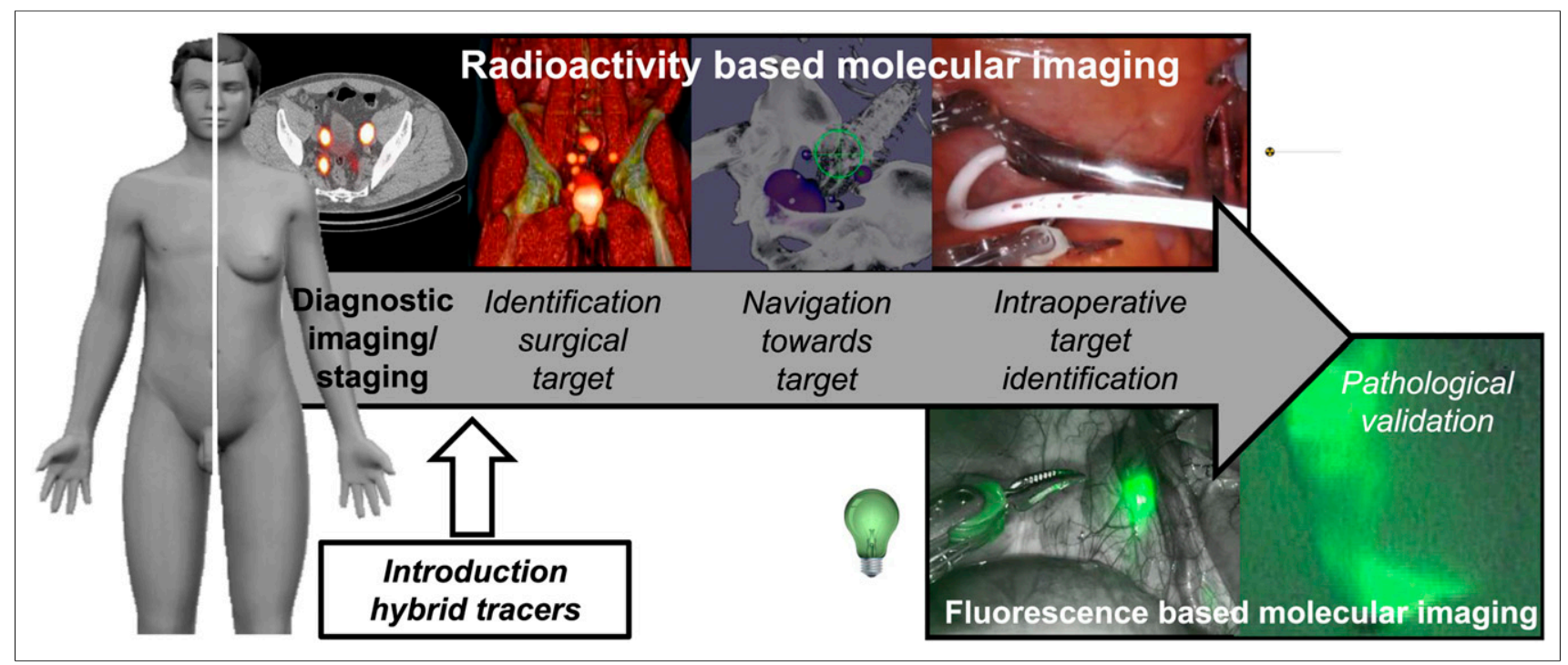

FIGURE 1. Schematic overview of concept of molecular imaging-guided precision surgery (example robot-assisted sentinel node procedure with hybrid tracer ICG-99mTc-nanocolloid).

\section{THE COMPETITIVE AND SYMBIOTIC ASPECTS OF RADIOGUIDED AND FLUORESCENCE- GUIDED SURGERY}

In the field of image-guided surgery, the hybrid concept coincides with monomodal radiotracers (radioguided surgery) and fluorescent dyes or tracers (fluorescence-guided surgery; Fig. 1). For some indications, clinical guidelines state the use of radioguidance or fluorescence-guidance approaches, but monomodal radiotracers and fluorescent tracers are also increasingly being implemented in experimental clinical trials, including cocktail formulations (1).

On the basis of the current scientific knowledge and technical possibilities, it seems worthwhile to critically assess the pros and cons of the use of hybrid versus monomodal radiotracers and fluorescent tracers (Fig. 2). Because of the comparably low tissue attenuation of $\gamma$-radiation and its high detection sensitivity, nuclear medicine has been able to use radiotracers for accurate noninvasive diagnostics. Especially, fused biochemical and anatomic information obtained through PET/CT, PET/MR, and SPECT/CT help place suspected pathologic conditions in their anatomic context (a road map) (6). Intraoperatively, real-time $\gamma$-probebased tracing is currently the method of choice in radioguided surgery; however, in some cases this method is also complemented by intraoperatively acquired static images obtained using, for example, a portable $\gamma$-camera or a freehand SPECT scan (7). Intraoperative radioguidance has been successfully applied in several targeted applications (Fig. 3), the most important ones being sentinel node biopsy (4), neuroendocrine tumors $(8)$, and recurrent prostate cancer (9). In these applications, the high detection sensitivity has made use of microdosing $(<100 \mu \mathrm{g} /$ patient $)$. Decay characteristics, relatively low tracer cost, and broad availability have made ${ }^{99 \mathrm{~m}} \mathrm{Tc}$ the radionuclide of choice for radioguided surgery $(9,10)$. However, although the spatial resolution of radioguidance modalities lies in the highmillimeter to low-centimeter range (7), the limited tissueinduced signal attenuation means lesion delineation can suffer from background signals coming from underlying tissues or organs.

Compared to radioguidance, optical guidance has a superior spatial resolution and is detectable even down to the microscopic level (5); Cerenkov light can provide a resolution of less than $2 \mathrm{~mm}(11,12)$. Unfortunately, light suffers heavily from tissue-induced signal attenuation, which limits the in-depth signal penetration of even nearinfrared dyes to less than $1 \mathrm{~cm}$ (13). While fluorescence imaging is sensitive in the lab (in vitro, ex vivo, and in mouse models), the aforementioned in vivo signal attenuation severely limits the detection sensitivity in human subjects (14). This statement is couched in the fact that fluorescence imaging in rodents is hardly predictive of clinical value. Despite these limitations, however, the major asset of fluorescence imaging is its capability to accommodate the surgeon's desire to optically identify the lesions in real time. In this context, Cerenkov imaging, with its lowluminescence brightness, represents an exception, since it requires the surgeon to still rely on static images (11). Routine clinical implementation of fluorescence-guided surgery has primarily been focused on physiologic indication, where flow information provides a valuable imaging readout (Fig. 3). At doses well above the microdosing level, fluorescent tracers such as fluorescein and ICG have allowed for superficial fluorescence guidance in dynamic processes such as vascular perfusion (angiography and anastomosis), lymphangiography (lymphatic drainage), liver clearance (cholecystectomies and identification of hepatobiliary metastases), and renal clearance (ureter visualization) (5). Metabolism of 5-ALA in lesions, however, provides an indirect targeted application for fluorescence imaging (5). 


\begin{tabular}{|c|c|c|c|c|c|}
\hline Tracer & Application & Parental tracer & $\begin{array}{c}\text { Year of first } \\
\text { clinical } \\
\text { implementation }\end{array}$ & $\begin{array}{l}\text { No. of } \\
\text { patients }\end{array}$ & $\begin{array}{l}\text { Clinical } \\
\text { routine? }\end{array}$ \\
\hline ICG-99mTc-nanocoloid $(4,19-21)$ & $\begin{array}{l}\text { Sentinel node imaging, } \\
\text { radioguided occult lesion } \\
\text { localization, tumor } \\
\text { margin delineation }\end{array}$ & $\begin{array}{l}\text { 99mTc-nanocolloid } \\
\text { (head-to-head } \\
\text { reproducibility } \\
\text { study performed } \\
\text { (28) }\end{array}$ & 2009 & $>1,500$ & Yes \\
\hline${ }^{131}$ I-fluorescein (3) & Brain tumor identification & Fluorescein & 1950 & 104 & No \\
\hline Cerenkov (18) & $\begin{array}{l}\text { Rectal cancer } \\
\text { identification, head } \\
\text { and neck } \\
\text { cancer, melanoma }\end{array}$ & ${ }^{18} \mathrm{~F}-\mathrm{FDG}$ & 2014 & $>60^{*}$ & No \\
\hline $\begin{array}{l}{ }^{111} \text { In-DOTA-girentuximab- } \\
\text { IRDye800CW (37) }\end{array}$ & $\begin{array}{l}\text { Clear cell renal cell } \\
\text { carcinoma identification }\end{array}$ & $\begin{array}{l}\text { PET tracer } \\
\text { girentuximab }\end{array}$ & 2018 & 15 & No \\
\hline${ }^{68} \mathrm{Ga}-I R D y e 800 \mathrm{CW}-\mathrm{BBN}$ (38) & $\begin{array}{l}\text { Glioblastoma multiforme } \\
\text { identification }\end{array}$ & Radiolabeled BBN & 2018 & 14 & No \\
\hline${ }^{125}$ I-methylene blue (17) & $\begin{array}{l}\text { Sentinel lymph node } \\
\text { biopsy; parathyroid } \\
\text { adenoma identification }\end{array}$ & Methylene blue & 2007 & 12 & No \\
\hline${ }^{123}$ I-methylene blue (2) & & & 1972 & 5 & No \\
\hline FITC-125I-CEA-mAb (39) & Colorectal carcinoma & $\begin{array}{l}\text { Radiolabeled } \\
\text { CEA-mAb }\end{array}$ & 1992 & 6 & No \\
\hline${ }^{124}$ I-cRGDY-PEG-C (40) & $\begin{array}{l}\text { Metastatic melanoma } \\
\text { identification }\end{array}$ & cRGDY-PEG-C ${ }^{\dagger}$ & 2014 & 5 & No \\
\hline
\end{tabular}

*Number was provided in oral communication with Jan Grimm during the World Molecular Imaging Congress 2019.

${ }^{\dagger}$ Not clinically used.

IRDye800CW = infrared dye 800CW; BBN = bombesin; FITC = fluorescein isothiocyanate; CEA = carcinoembryonic antigen; $\mathrm{mAb}=$ monoclonal antibody.

\section{EARLY CLINICAL IMPLEMENTATION OF HYBRID TRACERS}

Pioneering clinical trials with hybrid tracers $(15,16)$ have helped to overcome some reservations among researchers and surgeons with regard to this innovative surgical guidance concept. Table 1 illustrates that the field has moved forward over the years and underscores that different combinations of isotopes and dyes can be used in the clinical setting. Analogs such as ICG-99m Tc-nanocolloid (4), ${ }^{131} \mathrm{I}-$ fluorescein (3), ${ }^{123,125}$ I-methylene blue $(2,17)$, and Cerenkov imaging $(12,18)$ add to procedures previously performed with parental radiotracers or fluorescent tracers. Of all the hybrid tracers used in patients to date, ICG- ${ }^{99 \mathrm{~m}} \mathrm{Tc}$-nanocolloid is the most frequently and widely applied (4,19-21). A recent study with this hybrid tracer also indicated that fluorescence guidance without prior knowledge of the location of the lesion potentially results in incomplete resection (1).

\section{HYBRID TRACER DEVELOPMENT}

The literature on the preclinical use and assessment of hybrid tracers can be considered substantial. After the pioneering review by Culver et al. (22), numerous others have addressed the topic $(5,16,23-26)$. Besides oncologic imaging targets, such as human epidermal growth factor receptor 2, prostate-specific membrane antigen, carcinoembryonic antigen, $\alpha_{5} \beta_{3}$, somatostatin receptor 2 , and chemokine receptor 4 or sentinel node imaging, applications in, for example, infectious diseases have been reported. In preclinical studies, a plurality of imaging labels has been incorporated into hybrid tracer designs: radiolabels (e.g. ${ }^{99 \mathrm{~m} T c},{ }^{111} \mathrm{In},{ }^{18} \mathrm{~F}$, ${ }^{68} \mathrm{Ga}$, and ${ }^{89} \mathrm{Zr}$ ) and fluorescent labels (e.g., fluorescein isothiocyanate [FITC], cyanine dye CY5, 800CW, ZW800, and ICG). In an attempt to cover the essence of these initiatives, we refer to key review articles according to their chemical scaffold: peptides $(24,25)$, single-domain antibodies (26), antibodies $(16,24)$, and nanoparticles and bionanoparticles (5). From this combined literature we concluded that there are essentially 3 ways of creating a hybrid tracer: The first is functionalization of large proteins and nanoparticles, generally occurring with separate radiolabels and fluorescent labels. The second is functionalization of peptides, taking place with hybrid imaging labels that contain both a fluorescent label and a radiolabel or by functionalizing the $\mathrm{C}$ - and $\mathrm{N}$-terminal ends with a different imaging label. For the third, being small molecules, only hybrid imaging labels are an option. 


\section{Radioguided surgery}

Pros radioguided surgery:

Relatively small imaging labels

- Suitable radioisotopes with optimal $t_{1 / 2}$ and low energy available

- Low signal attenuation by tissue

- Allows non-invasive lesion detection in deep tissue layers $(>10 \mathrm{~cm})$

Ability to provide quantitative biodistribution 3D images provide a surgical roadmap

\section{Cons radioguided surgery:}

- Relatively poor imaging resolution

- Real-time detection only via acoustic tracing

- Intraoperative imaging feedback via static images

- Cannot serve as inherent "staining" for molecular pathological evaluation of tissue
Cons fluorescence guided surgery:

- Relatively large imaging labels

- Bleaching of fluorescent signal may occur

- Severe signal attenuation by tissue

- Only suitable for superficial imaging $(<1 \mathrm{~cm})$

- Not suitable for non-invasive (in depth) imaging

- Fluorescent signal intensity cannot be used to quantify the tracer distribution

Autofluorescence impacts specificity and sensitivity
Hybrid concept

("Best-of-both-worlds")

\section{Fluorescence guided surgery}

FIGURE 2. Pros and cons of radioguided and fluorescence-guided surgery.

\section{KEY FEATURES OF HYBRID TRACER DESIGN}

Although the addition of a radiolabel and a fluorescent dye to a targeting vector (e.g., small molecule, peptide, singledomain antibody, antibody, or nanoparticle) may seem trivial, generation of hybrid tracers suitable for clinical translation certainly represents a challenge. Here, some key design features can be taken into consideration and may support decision making (27).

Needless to say, successful chemical and technologic imaging innovations should address a specific clinical need. In doing so, incorporation of a complementary imaging signature should ideally help to realize a near-seamless extension of existing nuclear medicine diagnostics. Achieving this goal implies selection of known targets with proven clinical value-for example, high receptor overexpression as seen in neuroendocrine tumors or prostate-specific membrane antigen-positive lesions $(8,9)$. Most preclinical efforts in fluorescent or hybrid tracer design, however, do not benchmark their work to the current medical standard or a reference radiotracer. In the clinic, structurally optimized and clinically established radiotracers should be used to select patients who will then benefit from surgery. Only when surgery is scheduled may a second scan (with the hybrid tracer) be performed to ensure that the lesions targeted during surgery correspond with those defined on diagnostic imaging.
Since the chemical composition of a hybrid tracer intrinsically differs from that of the parental radiotracer, such a 2-step procedure also helps establish the reproducibility in tracer performance $(9,28)$. Here, the Cerenkov imaging concept holds a potential advantage because its optical signal is based on the parental PET tracer (12). The use of monolabeled fluorescent tracers for surgical guidance does not support such a critical reproducibility check and could mean that the surgical resection deviates from its planning, which was based on the road map defined by the diagnostic scan (l).

One of the key questions regarding the application of hybrid tracers revolves around the gap in sensitivity between radioactive and fluorescence imaging in vivo; radiotracers with a high specific activity can be used for imaging at a picomolar dose, whereas fluorescent agents are often used in the micromolar range. This sensitivity gap suggests that relatively high quantities of hybrid tracer are required and specific activity should be reduced in these formulations. Clinical use of the hybrid tracer ICG- ${ }^{99 \mathrm{~m} T c-}$ nanocolloid, however, indicates that the hybrid concept supports the use of much lower quantities of fluorescent dye (29). A study with ICG-99m Tc-nanocolloid (14) further implied that the microdosing concept may well be compatible with fluorescence-guided surgery, an observation that has been underlined by others $(12,30)$. The same study did, 


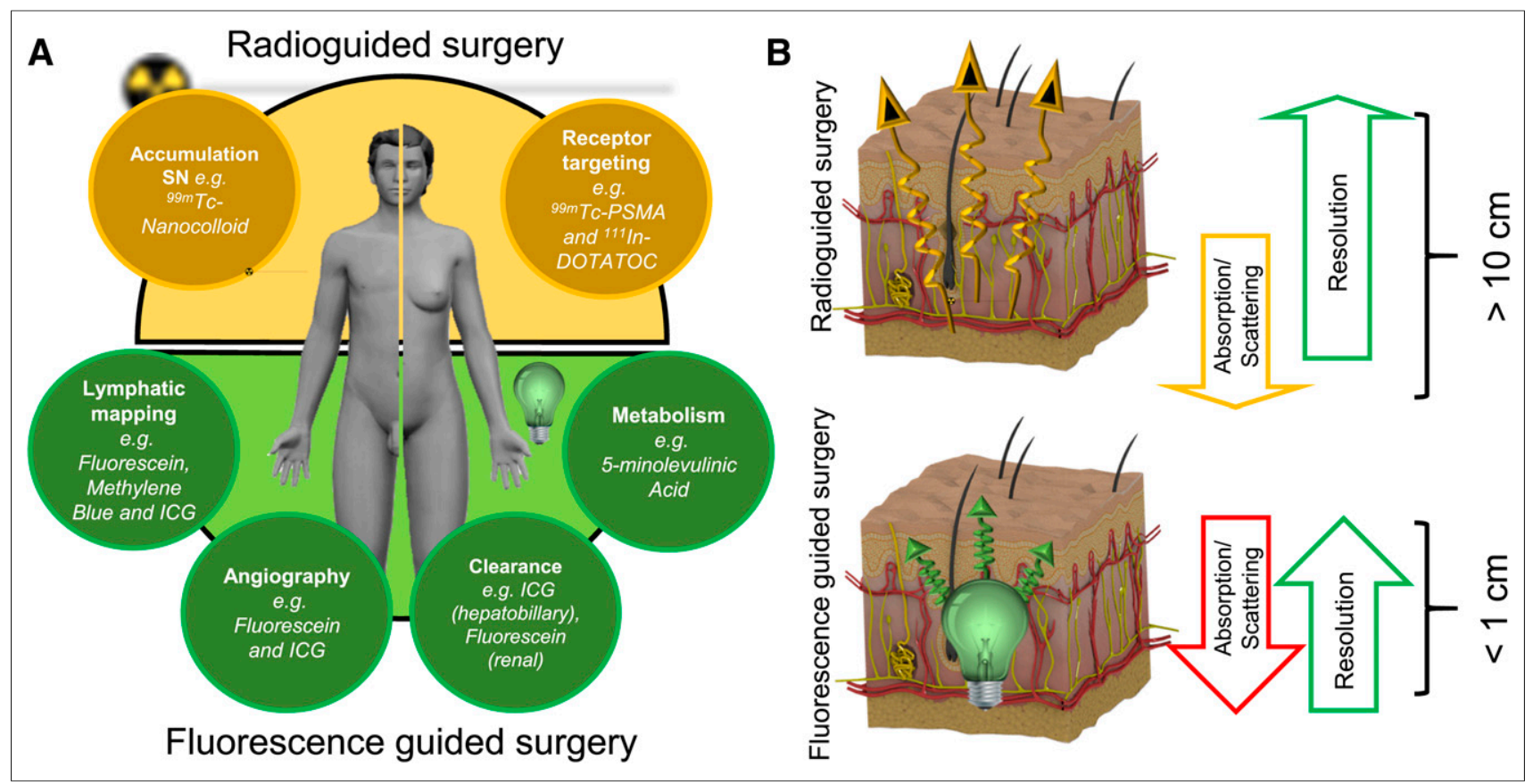

FIGURE 3. (A) Overview of routine indications for radioguided and fluorescence-guided surgery. (B) Signal penetration vs. resolution.

however, indicate that fluorescence-guidance became less accurate in areas with relatively low accumulation rates (14). This trend, combined with relatively low tumor receptor density expressions, could mean that fluorescence imaging will underestimate the number of lesions when not supported by nuclear medicine.

After saturation of all target sites in vivo, an increase in dose may negatively influence the specificity. Hence, the optical brightness of fluorescence needs to be improved to make it fully compatible with microdosing. Fluorescence signals can be amplified by increasing the excitation light intensity (11) or using dyes with a higher brightness (13). Given their low toxicity, low molecular weight $(<1,000$ $\mathrm{Da}$ ), and widespread clinical use, cyanine dyes are the dyes of choice for fluorescence-guided surgery. It is, however, unfortunate that the near-infrared CY7 analogs display a low brightness (31). Introduction of additional aromatic units on the indoles reduces the brightness further, whereas increasing the rigidity of the polymethine bridge enhances the brightness (32). Far-red CY5 analogs provide a substantially brighter alternative (13), which can be improved even further via dye sulfonation (33). This increase in brightness was shown to translate into a deeper signal penetration (13). Inorganic dyes such as quantum dots have been proposed as bright dyes for fluorescence-guided surgery. Unfortunately, this brightness comes at the cost of a large Stokes shift (possibly limiting tissue penetration of the excitation light), a relatively high molecular weight that influences dosing, and the presence of potentially toxic elements. In Cerenkov imaging, the brightness is directly related to the radioisotope used and is thus inherent to the radiotracer used (11).
As a rule of thumb, a signal-to-background ratio of more than 2 is essential for efficient surgical guidance using tracers, and thus specificity of tracer accumulation and well-balanced accumulation and clearance kinetics are key. It is known that the fluorescent dye component of hybrid tracers, even when large proteins are used as targeting vectors, influences their pharmacokinetics (e.g., via the lipophilicity of fluorescent dyes, which can influence serum binding and nonspecific interactions with nontarget tissue) and receptor affinity. In a direct comparison, Baranski et al. demonstrated that use of structurally very different fluorescent dye moieties in prostate-specific membrane antigen tracers affected the biodistribution (34), a finding that is underlined by studies that investigated how variation in cyanine dye can be used to tailor tracer performance $(13,35)$. For example, reducing the dye lipophilicity can positively affect tumor-to-background ratios, whereas increasing the number of charged moieties enhances renal uptake (13). Interestingly, these effects can vary between targeting moieties (33). Nevertheless, many chemists still adhere to a one-size-fits-all approach that is often based on the potential radiation and chemically unstable near-infrared dye $800 \mathrm{CW}(24,32)$. When inorganic nanoparticles are used as a fluorescent label, their sheer size relative to the targeting vectors means the dye properties dictate the in vivo kinetics (36).

\section{FUTURE PROSPECTS}

Although the field of fluorescence-guided surgery is growing, it is becoming increasingly apparent that some 


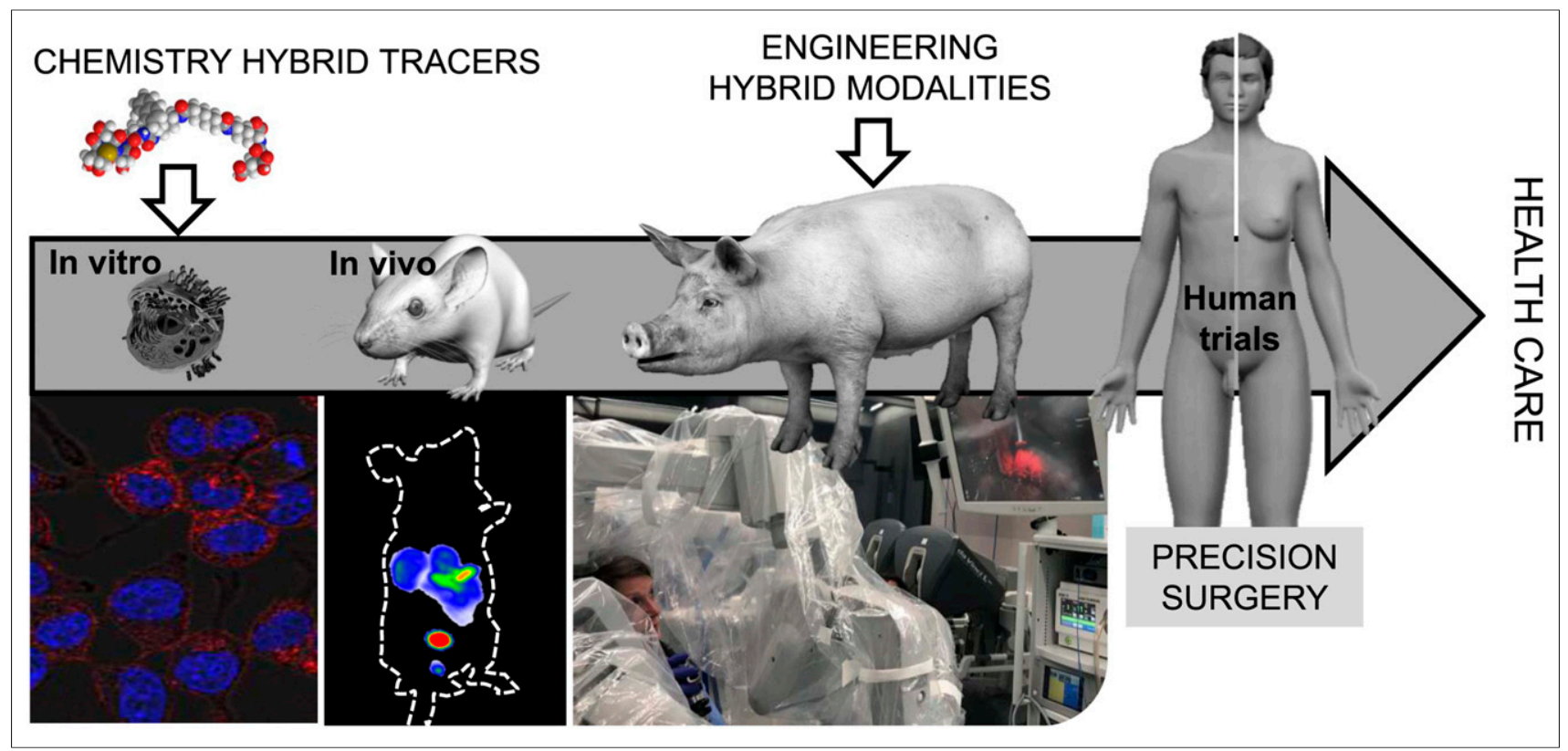

FIGURE 4. Translational pipeline toward routine health care for innovations in chemistry and engineering.

inherent limitations of the photophysics can be overcome by integrating fluorescence imaging with nuclear medicine. Although ongoing, clinical implementation of such concepts still remains restricted. One obvious discrepancy between clinical implementation and the more widespread preclinical technology development is that rodent models are not very representative of the clinical situation regarding, for example, dose, physical size, rate of excretion and metabolism, biodistribution, or tumor growth and composition. Although often ignored, these differences also affect the compatibility of tracers with clinically available imaging modalities. Hence, experiments on large animals (e.g., pig, veterinary patients, or disease models; Fig. 4) are required to efficiently engineer matching modalities such as hybrid modalities or GPS-like surgical navigation strategies that facilitate multiplexing of different signatures (7). By extending the hybrid concept into engineering (Fig. 4), the field is maturing and the chance to create a clinical impact is increasing.

\section{CONCLUSION}

The introduction of hybrid tracers has provided a valuable extension to the fields of molecular imaging, nuclear medicine, and, in particular, radioguided and fluorescenceguided surgery. Early clinical translations have already had an impact on clinical care. This impact helps shape a landscape that facilitates further development and translational efforts in the areas of chemistry and engineering, makes the hybrid tracer concept more accessible in the surgical and nuclear medicine environment, and supports the spread of the hybrid concept to clinicians and researchers in different surgical disciplines.

\section{DISCLOSURE}

No potential conflict of interest relevant to this article was reported.

\section{REFERENCES}

1. Meershoek P, Wit E, Buckle T, et al. Evaluation of the requirement of an integrated roadmap for identification of lesions in unknown locations using fluorescence-guided surgery in prostate cancer patients. J Nucl Med. November 11, 2019 [Epub ahead of print].

2. Blower PJKA, O'Doherty MJ, Collins RE, Coakley AJ. ${ }^{123}$ I-methylene blue: an unsatisfactory parathyroid imaging agent. Nucl Med Commun. 1992;13:522-527.

3. Moore GE, Kohl DA, Marvin JF, Wang JC, Caudill CM. Biophysical studies of methods utilizing fluorescein and its derivatives to diagnose brain tumors. Radiology. 1950;3:344-362.

4. KleinJan GH, van Werkhoven E, van den Berg NS, et al. The best of both worlds: a hybrid approach for optimal pre- and intraoperative identification of sentinel lymph nodes. Eur J Nucl Med Mol Imaging. 2018;45:1915-1925.

5. van Leeuwen FW, Hardwick JC, van Erkel AR. Luminescence-based imaging approaches in the field of interventional molecular imaging. Radiology. 2015;276:12-29.

6. Olmos RA, Vidal-Sicart S, Nieweg OE. SPECT-CT and real-time intraoperative imaging: new tools for sentinel node localization and radioguided surgery? Eur J Nucl Med Mol Imaging. 2009;36:1-5.

7. Van Oosterom MN, Rietbergen DDD, Welling MM, et al. Recent advances in nuclear and hybrid detection modalities for image-guided surgery. Expert Rev Med Devices. 2019;16:711-734.

8. Adams S, Baum RP. Intraoperative use of gamma-detecting probes to localize neuroendocrine tumors. $Q$ J Nucl Med. 2000;44:59-67.

9. Robu S, Schottelius M, Eiber M, et al. Preclinical evaluation and first patient application of ${ }^{99 \mathrm{~m}}$ Tc-PSMA-I\&S for SPECT imaging and radioguided surgery in prostate cancer. J Nucl Med. 2017;58:235-242.

10. Herrmann K, Nieweg OE, Povoski SP, eds. Radioguided Surgery: Current Applications and Innovation Directions in Clinical Practice. New York, NY: Springer; 2016:75-101.

11. Chin PT, Welling MM, Meskers SC, et al. Optical imaging as an expansion of nuclear medicine: Cerenkov-based luminescence vs fluorescence-based luminescence. Eur J Nucl Med Mol Imaging. 2013;40:1283-1291.

12. Shaffer TM, Pratt EC, Grimm J. Utilizing the power of Cerenkov light with nanotechnology. Nat Nanotechnol. 2017;12:106-117.

13. Buckle T, van Willigen DM, Spa SJ, et al. Tracers for fluorescence-guided surgery: how elongation of the polymethine chain in cyanine dyes alters the pharmacokinetics of a dual-modality c[RGDyK] tracer. J Nucl Med. 2018;59:986-992. 
14. KleinJan GH, Bunschoten A, van den Berg NS, et al. Fluorescence guided surgery and tracer-dose, fact or fiction? Eur J Nucl Med Mol Imaging. 2016;43:1857-1867.

15. van Leeuwen FW, Valdes-Olmos R, Buckle T, Vidal-Sicart S. Hybrid surgical guidance based on the integration of radionuclear and optical technologies. $\mathrm{Br} \mathrm{J}$ Radiol. 2016;89:20150797.

16. Hernandez Vargas S, Ghosh SC, Azhdarinia A. New developments in duallabeled molecular imaging agents. J Nucl Med. 2019;60:459-465.

17. Cundiff JD, Wang YZ, Espenan G, et al. A phase I/II trial of ${ }^{125} \mathrm{I}$ methylene blue for one-stage sentinel lymph node biopsy. Ann Surg. 2007;245:290-296.

18. Thorek DL, Riedl CC, Grimm J. Clinical Cerenkov luminescence imaging of ${ }^{18}$ F-FDG. J Nucl Med. 2014;55:95-98.

19. Christensen A, Charabi B, Mortensen J, Kiss K, Kjær A, von Buchwald C. Feasibility of real-time near-infrared fluorescence tracer imaging in sentinel node biopsy for oral cavity cancer patients. Ann Surg Oncol. 2016;23:565-572.

20. Paredes P, Vidal-Sicart S, Campos F, et al. Role of ICG- ${ }^{99 \mathrm{~m}} \mathrm{Tc}$-nanocolloid for sentinel lymph node detection in cervical cancer: a pilot study. Eur J Nucl Med Mol Imaging. 2017;44:1853-1861.

21. Stoffels I, Leyh J, Poppel T, Schadendorf D, Klode J. Evaluation of a radioactive and fluorescent hybrid tracer for sentinel lymph node biopsy in head and neck malignancies: prospective randomized clinical trial to compare ICG-99m Tc-nanocolloid hybrid tracer versus ${ }^{99 \mathrm{~m}}$ Tc-nanocolloid. Eur J Nucl Med Mol Imaging. 2015;42: 1631-1638.

22. Culver J, Akers W, Achilefu S. Multimodality molecular imaging with combined optical and SPECT/PET modalities. J Nucl Med. 2008;49:169-172.

23. Ahn SH, Boros E. Nuclear and optical bimodal imaging probes using sequential assembly: a perspective. Cancer Biother Radiopharm. 2018;33:308-315.

24. Azhdarinia A, Ghosh P, Ghosh S, Wilganowski N, Sevick-Muraca EM. Duallabeling strategies for nuclear and fluorescence molecular imaging: a review and analysis. Mol Imaging Biol. 2012;14:261-276.

25. Kuil J, Velders AH, van Leeuwen FW. Multimodal tumor-targeting peptides functionalized with both a radio- and a fluorescent label. Bioconjug Chem. 2010;21:1709-1719.

26. Lee SY, Jeon SI, Jung S, Chung IJ, Ahn CH. Targeted multimodal imaging modalities. Adv Drug Deliv Rev. 2014;76:60-78.

27. van Leeuwen FWB, Cornelissen B, Caobelli F, et al. Generation of fluorescently labeled tracers-which features influence the translational potential? EJNMMI Radiopharm Chem. 2017;2:15.

28. Brouwer OR, Buckle T, Vermeeren L, et al. Comparing the hybrid fluorescentradioactive tracer indocyanine green- ${ }^{99 \mathrm{~m}} \mathrm{Tc}$-nanocolloid with ${ }^{99 \mathrm{~m}} \mathrm{Tc}$-nanocolloid for sentinel node identification: a validation study using lymphoscintigraphy and SPECT/CT. J Nucl Med. 2012;53:1034-1040.

29. Schaafsma BE, Verbeek FP, Rietbergen DD, et al. Clinical trial of combined radio- and fluorescence-guided sentinel lymph node biopsy in breast cancer. $\mathrm{Br} \mathrm{J}$ Surg. 2013;100:1037-1044.

30. Lamberts LE, Koch M, de Jong JS, et al. Tumor-specific uptake of fluorescent bevacizumab-IRDye $800 \mathrm{CW}$ microdosing in patients with primary breast cancer: a phase I feasibility study. Clin Cancer Res. 2017;23:2730-2741.

31. Levitus M, Ranjit S. Cyanine dyes in biophysical research: the photophysics of polymethine fluorescent dyes in biomolecular environments. $Q$ Rev Biophys. 2011;44:123-151.

32. van der Wal SKJ. Valentijn ARPM, van Leeuwen FWB. Synthesis and systematic evaluation of symmetric sulfonated centrally $\mathrm{C}-\mathrm{C}$ bonded cyanine near-infrared dyes for protein labeling. Dyes Pigm. 2016;132:7-19.

33. Hensbergen AWB, van Willigen DM, Schottelius M, et al. Hybrid tracers based on cyanine backbones targeting prostate-specific membrane antigen: tuning pharmacokinetic properties and exploring dye-protein interaction. J Nucl Med. September 3, 2019 [Epub ahead of print].

34. Baranski AC, Schafer M, Bauder-Wust U, et al. PSMA-11-derived dual-labeled PSMA inhibitors for preoperative PET imaging and precise fluorescence-guided surgery of prostate cancer. $J$ Nucl Med. 2018;59:639-645.

35. Berezin MY, Guo K, Akers W, et al. Rational approach to select small peptide molecular probes labeled with fluorescent cyanine dyes for in vivo optical imaging. Biochemistry. 2011;50:2691-2700.

36. Choi HS, Frangioni JV. Nanoparticles for biomedical imaging: fundamentals of clinical translation. Mol Imaging. 2010;9:291-310.

37. Hekman MC, Rijpkema M, Muselaers $\mathrm{CH}$, et al. Tumor-targeted dual-modality imaging to improve intraoperative visualization of clear cell renal cell carcinoma: a first in man study. Theranostics. 2018;8:2161-2170.

38. Li D, Zhang J, Chi C, et al. First-in-human study of PET and optical dual-modality image-guided surgery in glioblastoma using ${ }^{68} \mathrm{Ga}$-IRDye800CW-BBN. Theranostics. 2018;8:2508-2520.

39. Folli S, Wagnieres G, Pelegrin A, et al. Immunophotodiagnosis of colon carcinomas in patients injected with fluoresceinated chimeric antibodies against carcinoembryonic antigen. Proc Natl Acad Sci USA. 1992;89:7973-7977.

40. Phillips E, Penate-Medina O, Zanzonico PB, et al. Clinical translation of an ultrasmall inorganic optical-PET imaging nanoparticle probe. Sci Transl Med. 2014;6: $260 \mathrm{ra} 149$. 